\title{
Magnetic susceptibility anisotropy of myocardium imaged by cardiovascular magnetic resonance reflects the anisotropy of myocardial filament a-helix polypeptide bonds
}

Russell Dibb ${ }^{1,2}$, Yi Qi ${ }^{1}$ and Chunlei Liu ${ }^{3,4^{*}}$

\begin{abstract}
Background: A key component of evaluating myocardial tissue function is the assessment of myofiber organization and structure. Studies suggest that striated muscle fibers are magnetically anisotropic, which, if measurable in the heart, may provide a tool to assess myocardial microstructure and function.

Methods: To determine whether this weak anisotropy is observable and spatially quantifiable with cardiovascular magnetic resonance (CMR), both gradient-echo and diffusion-weighted data were collected from intact mouse heart specimens at 9.4 Tesla. Susceptibility anisotropy was experimentally calculated using a voxelwise analysis of myocardial tissue susceptibility as a function of myofiber angle. A myocardial tissue simulation was developed to evaluate the role of the known diamagnetic anisotropy of the peptide bond in the observed susceptibility contrast.

Results: The CMR data revealed that myocardial tissue fibers that were parallel and perpendicular to the magnetic field direction appeared relatively paramagnetic and diamagnetic, respectively. A linear relationship was found between the magnetic susceptibility of the myocardial tissue and the squared sine of the myofiber angle with respect to the field direction. The multi-filament model simulation yielded susceptibility anisotropy values that reflected those found in the experimental data, and were consistent that this anisotropy decreased as the echo time increased.

Conclusions: Though other sources of susceptibility anisotropy in myocardium may exist, the arrangement of peptide bonds in the myofilaments is a significant, and likely the most dominant source of susceptibility anisotropy. This anisotropy can be further exploited to probe the integrity and organization of myofibers in both healthy and diseased heart tissue.
\end{abstract}

Keywords: Anisotropic magnetic susceptibility, Myocardial fiber mapping, Multi-compartment relaxation, Resonance frequency shift

\footnotetext{
* Correspondence: chunlei.liu@duke.edu

${ }^{3}$ Brain Imaging \& Analysis Center, Duke University Medical Center, Box 3918, Durham, NC 27710, USA

${ }^{4}$ Radiology, Duke University Medical Center, Box 3808, Durham, NC 27710

USA

Full list of author information is available at the end of the article
} 


\section{Background}

Magnetic susceptibility describes the extent to which a substance becomes magnetized when placed in an external magnetic field. Many biological tissues exhibit either positive or negative susceptibility, and are termed paramagnetic or diamagnetic, respectively. Some of these tissues also have susceptibilities that are dependent on tissue orientation. In particular, striated muscle tissue is known to exhibit anisotropic magnetic susceptibility. Earlier studies have detected this susceptibility anisotropy in excised rabbit psoas muscle tissue suspended in a magnetic field [1] and in polymerized actin extracted from acetone powders of muscle tissue [2]. However, it is unknown if this anisotropy remains observable in bulk myocardium in an intact heart where the microstructures and molecular compositions are far more complex. Determining this anisotropy in an intact heart requires spatially localized susceptibility measurements, for which techniques relying on birefringence or superconducting quantum interference devices are limited.

Verifying the susceptibility anisotropy of myocardium and its underlying mechanisms using cardiovascular magnetic resonance (CMR) may lead to improved techniques for examining the microstructure of the heart. Myocardial fiber organization and structure are important determinants of myocardial stress and strain [3], are altered by cardiac hypertrophy [4] and infarction $[5,6]$, and are thought to play an important role in arrhythmogenesis [7]. Hence, mapping myofiber organization is important for assessing the functional properties of healthy and diseased hearts. Most commonly, ex vivo myocardial fiber mapping is carried out through diffusion tensor imaging (DTI) and histology [8-10]. Though a non-destructive imaging modality, cardiac DTI is challenged by spatial resolution limits and long scan times imposed by low signal-to-noise ratio (SNR), especially in the absence of contrast agent enhancement. On the other hand, histological techniques can yield wholeheart myofiber maps with very high spatial resolution, but are labor-intensive and require destruction of the organ. Alternatively, $\mathrm{T}_{2}{ }^{*}$-weighted gradient-recalled echo (GRE) CMR has shown potential as a method to visualize the microstructure of beating, isolated rat hearts [11]. In addition, GRE image phase is sensitive to changes in the magnetic field caused by magnetically susceptible components in tissues, such as deoxyhemoglobin, deoxymyoglobin, or calcification, and can be used for determining the susceptibility differences among tissues [12]. Considering the high-resolution capability of GRE phase and recent developments in susceptibility tensor imaging (STI) [13, 14], using CMR to image the susceptibility anisotropy of the heart may aid in assessing myocardial fiber integrity and alterations induced by cardiac diseases and disorders.
We present here a non-destructive method for imaging and quantifying the magnetic susceptibility anisotropy of whole myocardium in situ using CMR. The method, which details the relationship between apparent magnetic susceptibility and myofiber orientation, reveals that magnetic susceptibility anisotropy is extensive within the myocardium and not merely present in select tissue regions. Similar tools have recently been used to investigate anisotropic magnetic susceptibility in brain white matter [13, 15] and kidney tubules [16]. We hypothesize that the observed bulk magnetic susceptibility anisotropy of the heart originates from the $\alpha$-helix polypeptides that are prevalent in myocardial filaments. We show by comparison of experimental and simulated results that the structurally organized [17] and diamagnetically anisotropic $[18,19]$ bonds forming these molecules are potentially the chief sources of the observed widespread anisotropy.

\section{Methods}

\section{Animal model}

All animal preparation protocols were approved by the Duke University Institutional Animal Care and Use Committee. Four adult, male C57BL/6 mice (Charles River Labs, Raleigh, NC) were anesthetized with pentobarbital (Nembutal, Lundbeck Inc., Deerfield, IL), and a catheter was inserted into the right jugular vein. Using a peristaltic pump, each animal was perfused first with $0.2 \%$ heparin (1000 usp units/ml, Sagent Pharmaceuticals, Schaumburg, IL) in $0.9 \%$ saline solution at a rate of $8 \mathrm{ml} / \mathrm{min}$ for $5 \mathrm{~min}$. When perfusion began, the inferior vena cava and descending thoracic aorta were incised, allowing the blood to clear from the thorax, upper extremities and head. Next, the tissue was fixed using $150 \mathrm{ml}$ of $10 \%$ buffered formalin phosphate (SF 100-20, Fisher Scientific, Pittsburgh, PA) at a rate of $8 \mathrm{ml} / \mathrm{min}$. Finally, to preserve the shape of the heart [20], the specimen was perfused with $1.3 \%$ agarose gel (A9414-25G, Sigma-Aldrich, St. Louis, MO) at a rate of $8 \mathrm{ml} / \mathrm{min}$ for $2.5 \mathrm{~min}$. The gel was allowed to solidify within the chambers of the heart for $25 \mathrm{~min}$. The heart was then removed from the animal and stored for three days in $10 \mathrm{mM}$ phosphate-buffered saline $(\mathrm{pH} 7.4$, SigmaAldrich P-3813) prior to scanning.

\section{CMR microscopy}

CMR experiments were performed using a 9.4 T (400 MHz) 8.9-cm vertical bore Oxford magnet controlled by an Agilent VnmrJ 4.0 console. Each myocardium specimen was firmly affixed in an 11-mm cylindrical polyethylene cartridge filled with Galden ${ }^{\circ}$ (perfluoropolyether; Solvay Specialty Polymers) to provide a dark background in the images and mitigate tissue dehydration and susceptibility distortions at the 
specimen surface. In order to verify the presence of susceptibility anisotropy in localized myocardial regions, one heart specimen cartridge was placed inside a sphere, allowing for an arbitrary specimen orientation inside the coil. The coil apparatus [16] supported a solenoid radiofrequency resonator (21- $\mathrm{mm}$ diameter; 21-mm length). Magnitude and phase data were acquired using a GRE sequence with 16 echoes $\left(\mathrm{TE}_{1} /\right.$ $\Delta \mathrm{TE} / \mathrm{TE}_{16}=2.2 / 4.2 / 65.2 \mathrm{~ms}, \mathrm{TR}=150 \mathrm{~ms}, \alpha=35^{\circ}$, array size $=400 \times 300 \times 300$, isotropic voxel size $=45 \mu \mathrm{m}$, total scan time per orientation $=3.8 \mathrm{~h}$ ). Prior to every image acquisition, the myocardium specimen was repositioned in a new orientation with respect to the magnetic field. Twelve image orientations were acquired for this particular specimen. To assess susceptibility anisotropy without the need to reorient the specimen, the other three heart specimens were scanned in a smaller, $12 \mathrm{~mm} \times 25 \mathrm{~mm}$ (diameter $\times$ length) solenoid radiofrequency coil with the long axis of the myocardium fixed perpendicular to the main magnetic field direction. $\mathrm{T}_{1}$ recovery was measured in one of these specimens by acquiring image data from a central slice of the heart using a series of spin echo (SE) scans $(\mathrm{TE}=10 \mathrm{~ms} ; \mathrm{TR}=$ $20,40,80,160,320,640,1280$, and $2560 \mathrm{~ms}$; array size $=128 \times 128$; resolution $=90 \mu \mathrm{m})$. MR magnitude and phase image data were acquired from each of the three specimens using a 3-D spoiled GRE sequence with 16 echoes $\left(\mathrm{TE}_{1} / \Delta \mathrm{TE} / \mathrm{TE}_{16}=1.7 / 3.0 / 46.7 \mathrm{~ms}, \quad \mathrm{TR}=\right.$ $200 \mathrm{~ms}, \alpha=35^{\circ}$, array size $=256 \times 256 \times 256$, isotropic voxel size $=45 \mu \mathrm{m}$, total scan time $=3.6 \mathrm{~h}$ ). Diffusion tensor data were also acquired for all four individual specimens using one SE scan with $b=0 \mathrm{~s} / \mathrm{mm}^{2}$ and 12 diffusion-encoded SE scans with diffusion time $=$ $5.5 \mathrm{~ms}$, pulse separation $=17.0 \mathrm{~ms}$, and $\mathrm{b}=1850 \mathrm{~s} / \mathrm{mm}^{2}$ $(\mathrm{TE}=23.6 \mathrm{~ms}, \mathrm{TR}=2000 \mathrm{~ms}$, array size $=64 \times 64 \times 64$, isotropic voxel size $=180 \mu \mathrm{m}$ total scan time $=29.6 \mathrm{~h}$ ). Due to the long $\mathrm{T}_{1}$ of tissue, the DTI protocol required the acquisition of a smaller array to achieve adequate signal-to-noise ratio and prevent overly long scan times.

\section{MR data reconstruction and processing}

To extract the local susceptibility information from CMR images, the phase data must be appropriately processed to remove phase wraps as well as phase from sources outside the specimen. Thus, all of the multi-echo GRE image phase data were processed using an integrated Laplacian-based phase unwrapping and background phase removal algorithm, HARPERELLA [21]. With this processed phase information, the local susceptibility, $\mathbf{X}$, can then be approximated as a scalar-valued quantity by inverting the susceptibilityphase relationship in the laboratory frame of reference $[13,22]$,

$$
\phi=\mathrm{FT}^{-1}\left\{\left(\frac{1}{3}-\frac{k_{3}^{2}}{\mathbf{k}^{\mathrm{T}} \mathbf{k}}\right) \operatorname{FT}\left\{\mathrm{X}_{33}\right\}\right\} \gamma \mu_{0} H T E
$$

where $\phi$ is the processed image phase, FT and $\mathrm{FT}^{-1}$ are the forward and inverse Fourier transforms, $\mathbf{k}$ is the spatial frequency vector, $\chi_{33}$ is the last term in the $3 \times 3$ magnetic susceptibility tensor, $\gamma$ is the gyromagnetic ratio, $\mu_{0}$ is the vacuum permeability, $H$ is the applied magnetic field strength, and TE is the echo time. Note that the $X_{13}$ and $X_{23}$ terms have been ignored in deriving Equation 1 assuming they are much smaller than $X_{33}$ [13]. This inversion problem is ill posed because the dipole kernel in Equation 1 is equal to zero when $3 k_{3}^{2}-$ $\mathbf{k}^{\mathbf{T}} \mathbf{k}=0$. Several susceptibility mapping approaches have been formulated to overcome this challenge, including $\mathrm{k}$-space thresholding [23], multiple-orientation sampling [24], nonlinear regularization [25], compressed-sensing estimation [26], and first-order derivative approximation [27]. In this study, the susceptibility maps were iteratively calculated using the LSQR method [27] because this algorithm computes susceptibility maps efficiently, requires only one sampling orientation, and offers a high quality solution. After the susceptibility maps were calculated for each individual echo, an average susceptibility map was calculated for each specimen from the multi-echo susceptibility data.

In order to match the GRE image data resolution of each specimen, the diffusion-weighted image data were either resampled to a $256^{3}$ array for the singleorientation data ( $n=3$ specimens), or a $400 \times 300 \times 300$ array for the multi-orientation data $(n=1$ specimen). A diffusion tensor map for each specimen was then estimated using Diffusion Toolkit [28]. A myofiber angle map was determined by calculating the angle between the major eigenvector of the diffusion tensor and the magnetic field direction vector. Lastly, tissue relaxation parameters were estimated for the single-orientation image data. A voxelwise calculation of the $T_{1}$ relaxation time was made by fitting a longitudinal magnetization recovery curve to the signal from the $2 \mathrm{D}$ SE image data acquired using several TRs. The signal from each voxel in the multi-echo GRE magnitude image volumes was fit with a biexponential $\mathrm{T}_{2}{ }^{*}$ decay function, $\mathrm{S}(\mathrm{t})=\mathrm{S}_{\text {noise }}+$ $\mathrm{S}_{0, \mathrm{GRE}}\left[\mathrm{V}_{i} \times \exp \left(-\mathrm{t} / \mathrm{T}_{2 i} i^{*}\right)+\mathrm{V}_{e} \times \exp \left(-\mathrm{t} / \mathrm{T}_{2 e^{*}}\right)\right]$, where $\mathrm{S}_{\text {noise }}$ is the noise floor of the magnitude signal and $\mathrm{S}_{0, \mathrm{GRE}}$ is the signal at TE $=0 \mathrm{~ms}$, assuming that the proton densities of the two signal pools are similar. This was done in order to acquire the intracellular and extracellular volume fraction $\left(\mathrm{V}_{i}\right.$ and $\left.\mathrm{V}_{e}\right)$, as well as the intracellular and extracellular $\mathrm{T}_{2}{ }^{*}$. Using the mean $(n=3)$ volume fractions from the $\mathrm{T}_{2}$ * decay fit, the signal data from the $2 \mathrm{D}$ image series of longitudinal magnetization recovery measurements were fit to the curve, $S(t)=$ 
$\mathrm{S}_{0, \mathrm{SE}}\left[\mathrm{V}_{i}\left(1-\exp \left(-\mathrm{t} / \mathrm{T}_{1 i}{ }^{*}\right)\right)+\mathrm{V}_{e}\left(1-\exp \left(-\mathrm{t} / \mathrm{T}_{1 e^{*}}\right)\right)\right]$, subject to the constraint $\left(\mathrm{V}_{i}+\mathrm{V}_{e}\right) / \mathrm{T}_{1}=\mathrm{V}_{i} / \mathrm{T}_{1 i}+\mathrm{V}_{e} / \mathrm{T}_{1 e}$. The peak values of the intra- and extracellular $\mathrm{T}_{1}$ distributions were used as rough estimates of the intra- and extracellular $\mathrm{T}_{1}$ relaxation times. Signal fitting was performed using the "fit" function in MATLAB R2014a (MathWorks, Natick, MA). All other computations were performed using MATLAB unless otherwise specified.

\section{Quantitative analysis of tissue susceptibility as a function of fiber angle}

The multi-orientation GRE data were analyzed to determine the orientation dependence of magnetic susceptibility in myocardial tissue. First, all 12 GRE image volumes were registered to the corresponding diffusion tensor data. Three small ( $n=10$ pixels) regions of interest (ROIs) were manually selected from a 2-D image slice of the DTI data using the ITK-SNAP software [29]. Within each individual ROI were tissue voxels with similarly oriented myofibers, and the mean fiber direction of each ROI was approximately orthogonal to that of the two other ROIs. For each of the twelve registered GRE volume images, the mean apparent magnetic susceptibility of each ROI was related to the mean squared sine of the fiber angle with respect to the magnetic field. A linear regression model was then fit to the data in order to calculate the susceptibility anisotropy of myocardium.

Ideally, magnetic susceptibility anisotropy would be measured without the need to acquire data at multiple specimen orientations. For this reason, the correlation between susceptibility and myofiber orientation in the single-orientation image data was also studied. One large, 3D ROI was selected in each of the three remaining heart specimens. These ROIs included myocardial tissue but excluded the heart chambers as well as vessels that were large enough to segment. A voxelwise analysis then related the mean magnetic susceptibility of the multi-echo image data to the squared sine of the myocardial fiber angle in each specimen. Linear regression was again performed to measure the susceptibility anisotropy. Only voxels with an effective DTI fractional anisotropy above 0.3 and an intracellular voxel fraction, $\mathrm{V}_{i}$, within the middle $50 \%$ of values were examined to ensure that the results included coherently oriented myofibers and excluded small vessels.

\section{A molecular and multi-filament model for susceptibility anisotropy}

A myocardial tissue model was developed to evaluate the contribution of the diamagnetic anisotropy of the peptide bonds in myofilament proteins to the orientationdependent magnetic susceptibility observed in the rodent heart. Within a sarcomere is an organized lattice of thick and thin myofilaments. These filaments are primarily constructed out of the proteins myosin, tropomyosin, and actin. Both myosin and tropomyosin predominantly contain polypeptide chains in the $\alpha$-helix form, which is known to be diamagnetically anisotropic due to the structure of its peptide bonds [18]. In myosin, two $\alpha$-helices wind together, forming a coiled-coil structure. This myosin "tail" then twists together with several other myosin tails to form the thick filament. Like the myosin tail, tropomyosin is a coiled-coil protein, with two tropomyosin strands running along the thin filament. The other major component of the thin filament is actin, which contains about $40 \% \alpha$-helix [30]. Within the A-band of the sarcomere, overlapping myosin, tropomyosin, and actin filaments are oriented parallel to each other and to the long axis of the myofiber.

A section of sarcomere A-band was simulated inside a $69.3 \times 40.0 \times 42.9 \mathrm{~nm}$ volume and was divided into a $462 \times 266 \times 286$ voxel array with $0.15 \mathrm{~nm}$ isotropic resolution. This resolution was selected because it is the

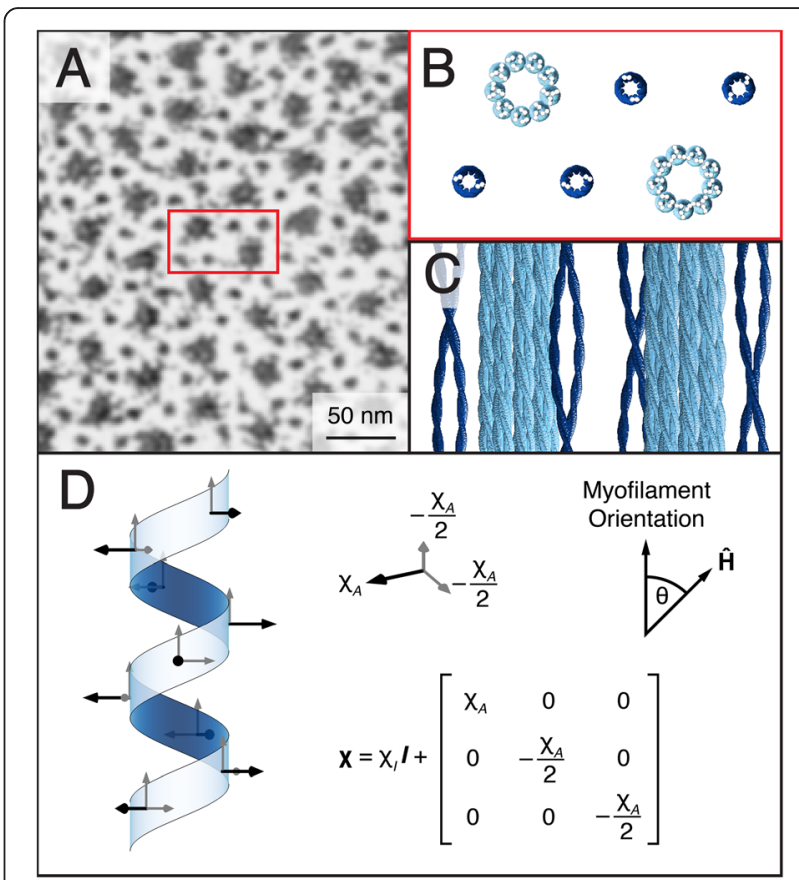

Fig. 1 Microstructural and mathematical basis of the myofilament model. a An electron microscope image of a cross-section of the sarcomere highlights the myofilament lattice in myocardial tissue (image courtesy of Margaret Goldstein, PhD, Baylor College of Medicine and Robert Perz-Edwards, PhD, Duke University). b A cross-sectional rendering of the myofilament model volume represents a unit that repeats throughout the sarcomere. c Thick (light blue) and thin (dark blue) filaments are represented in the model volume by secondary and tertiary a-helical structures. The F-actin is not represented in the model volume due to the less coherent organization of a-helices within actin subunits. $\mathbf{d}$ Bond locations and susceptibility tensors are defined according to the molecular structure of the a-helix. There is an average of 3.6 peptide bonds per helix repeat 
Table 1 Microstructural parameters defining the myofilament lattice in the model volume

\begin{tabular}{lcl}
\hline Microstructural Feature & Diameter, $\mathrm{nm}$ & Pitch, nm \\
\hline Thick filament & $16.0[32]$ & - \\
Thick subfilament & $4.0[32]$ & $42.9[61]$ \\
Myosin tail & $2.0[62]$ & $4.3[63]$ \\
Thin filament & $7.0[64]$ & $77.0[65]$ \\
Tropomyosin tail & $2.0[66]$ & $3.7[65]$ \\
a-helix & $1.0[67]$ & $0.54[67]$ \\
\hline
\end{tabular}

axial spacing between peptides in the $\alpha$-helix and is similar to the length of the diamagnetically anisotropic peptide bond $(0.132 \mathrm{~nm})$ [17]. Two thick and four thin filaments were simulated in the volume according to their $\alpha$-helical, secondary, and tertiary structures [31, 32], and then arranged to mimic the lattice structure of the smallest repeatable unit of the sarcomere (Fig. 1a-c). The simulated volume maintains the 2:1 thin-to-thick filament ratio observed in vertebrate myocardium with thick and thin filaments coaxially spaced at 40.0 and $23.1 \mathrm{~nm}$, respectively [33]. Due to the less coherent organization of the $\alpha$-helices in actin subunits [34], only myosin and tropomyosin $\alpha$-helices are represented in the volume (Fig. 1b-c). Finally, the peptide bond locations were simulated in the volume according to the pitch and spacing of the $\alpha$-helix polypeptide backbone. Table 1 details the literature-derived myofilament microstructural parameters used in the simulation.

When amino acids are arranged in an $\alpha$-helix, the peptide bonds all lie in a plane parallel to the helix axis (Fig. 1d). The magnetic susceptibility normal to this plane is defined as the anisotropic susceptibility component, $X_{A}$. For the purposes of this model, the in-plane susceptibilities are approximately equal, i.e., the susceptibility tensor is axially symmetric about the out-of-plane axis of the bond. This assumption is made because formamide, a simple prototype of a peptide linkage [35], is almost axially symmetric with respect to its magnetic susceptibility components about the out-of-plane (and most diamagnetic) axis [36]. Each voxel within the volume that contains a peptide bond was assigned the same relative susceptibility tensor, $\mathrm{X}$, reflecting the axial magnetic susceptibility symmetry of the peptide group (Fig. 1d). Each of these tensors was then rotated according to its position in the $\alpha$-helix. Using the theoretical molar susceptibility anisotropy value calculated by Pauling, $-5.36 \times 10^{-6} \mathrm{~cm}^{3} / \mathrm{mol}$ (CGS units) [19], and the volume of a simulated voxel, the model-specific volume susceptibility anisotropy of each voxel containing a peptide group was calculated as $\Delta \mathrm{X}=\mathrm{X}_{11}-\left(\mathrm{X}_{22}+\mathrm{X}_{33}\right) / 2=$ $-33.14 \mathrm{ppm}$ (SI units). From this value, the anisotropic susceptibility component is then $X_{A}=2 \Delta \mathrm{X} / 3=$
$-22.09 \mathrm{ppm}$. The isotropic susceptibility value of the peptide group, $X_{I}$, is not fixed as it is evaluated relative to the reference susceptibility. All other voxels in the simulated volume were assigned a relative susceptibility tensor with value $\mathbf{0}$.

With the axes of the simulated fibers oriented at an angle $(\theta)$ to the direction of the static field $(\hat{\mathbf{H}})$, threedimensional frequency maps for the model array $\left(f_{\text {array }}\right)$ were simulated using the tensor formulation of the susceptibility-frequency equation in the subject frame of reference [13],

$$
f_{\text {array }}=\mathrm{FT}^{-1}\left\{\left(\frac{\frac{1}{3} \mathbf{H} \mathbf{T F T}\{\mathbf{X}\} \mathbf{H}-\mathbf{H} \mathbf{T k k}^{\mathrm{T}} \mathrm{FT}\{\mathbf{X}\} \mathbf{H}}{k^{2}}\right) \operatorname{FT}\{\mathbf{x}\}\right\} \gamma \mu_{0} H
$$

Finally, a complex average of uniformly distributed spins across the entire 3D frequency map yielded a single, complex-valued signal to represent the intracellular volume. In contrast, the extracellular volume was assigned a relative susceptibility tensor value of $\mathbf{0}$, which is the reference susceptibility. As a result, the net signal of the extracellular space has zero phase.

Within the myocardium are two anatomical compartments with distinct signal contributions: intracellular and extracellular [37]. To simulate a model susceptibility value for a volume the size of a voxel in the experimental data, the complex-valued signal from the myofilament lattice in the intracellular compartment must be appropriately combined with the signal from the extracellular compartment. In this model, the magnetic resonance signal frequency shift, $\Delta f$, generated by a myocardial tissue voxel is the result of a weighted average:

$$
\Delta f=\angle\left\{\sum_{j=i, e} S_{j} \rho_{j} V_{j} e^{-T E / T_{2_{j}}^{*}} \frac{\left(1-e^{-T R / T_{1_{j}}}\right) \sin \alpha}{1-e^{-T R / T_{1_{j}}} \cos \alpha}\right\} /-2 \pi T E
$$

Here, the subscripts $i$ and $e$ represent the intra- and extracellular compartments, respectively; $S$ is the complex-valued signal; $\rho$ is the relative spin density, which the model assumes is the same for each compartment for simplicity since the literature values for the water content of perfused rodent hearts varies by study [38]; and $\alpha$ is the flip angle. According to Equation 3, the model applies $\mathrm{T}_{1}$ and $\mathrm{T}_{2}{ }^{*}$ weighting to the intra- and extracellular compartments, but also takes into account the intracellular $\mathrm{T}_{2}$ ' decay that is inherently simulated by the model due to the inhomogeneous magnetic susceptibility distribution of the intracellular volume.

An estimate of the bulk susceptibility of a voxel containing myocardial tissue, $X$, was then calculated using the theoretical relationship $\Delta f / f_{0}=\mathrm{X} / 3$ with the 
correction of spherical inclusion [39], where $f_{0}$ is the resonance frequency. The simulation was then repeated for a range of myocardial fiber orientations $\left(0-90^{\circ}\right)$ with respect to $\hat{\mathbf{H}}$ to model susceptibility anisotropy as a function of TE. The isotropic susceptibility value of the peptide bond tensor, $X_{I}$, was calculated by the model to fit the specimen susceptibility data (which is a relative measure and not absolute) using a mean squared error minimizing algorithm that was weighted by the inverse of the standard deviation of the experimental data (step size $=0.01 \mathrm{ppm}$ ). With $\chi_{I}$ calculated, the simulation was then repeated while excluding the extracellular space from the model in order to estimate the volume susceptibility anisotropy of the myofilament lattice. The thick and thin filaments were then simulated separately to ascertain the relative contributions of each structure to the tissue susceptibility anisotropy. Variations of this model were simulated to analyze the impact of implementing multi-compartment signal relaxation on the model volume's bulk susceptibility anisotropy. The first scenario involved omitting $T_{1}$, and then both $T_{1}$ and $T_{2}{ }^{*}$ relaxation from the model. In the second scenario, the $\mathrm{T}_{2}{ }^{*}$ weighting was adjusted by superposing $\mathrm{T}_{2}, \mathrm{~T}_{2}{ }^{*}$ and no additional weighting on top of the inherent intracellular $\mathrm{T}_{2}$ ' that exists due to the field inhomogeneity produced by the simulated peptide bonds.

\section{Results}

\section{Orientation-dependent susceptibility contrast}

The results of the phase processing pipeline for a single echo image from a typical myocardium specimen are shown in Fig. 2. Since the gel-filled regions of the heart have a tendency to obfuscate image properties exhibited by myocardial tissue (Fig. 2b-c), the chambers of the heart have been masked out for display in the figures that follow. The relationship between the mean magnetic susceptibility contrast and the mean myofiber orientation for the multi-orientation GRE data is shown in
Fig. 3. Linear regression reveals a negative trend in each of the three small ROIs, though the degree of the susceptibility anisotropy and the coefficient of determination vary. The correlation is strongest in the papillary muscles (red), which, not surprisingly, also have very strong DTI fractional anisotropy. The relationship between the mean magnetic susceptibility contrast of the multi-echo GRE image data acquired with a singleorientation and the DTI-based myofiber orientation of the tissue is illustrated for a typical specimen in Fig. 4. As seen in the mean susceptibility (Fig. $4 \mathrm{~b}$ ) and myofiber angle (Fig. 4d) maps, myofibers perpendicular to the field appear diamagnetic, whereas those parallel to the field appear paramagnetic relative to the reference susceptibility.

The results of the biexponential signal-fitting algorithm from a typical heart specimen are shown in Fig. 5. Histograms detailing the distribution of volume fractions and $\mathrm{T}_{2}{ }^{*}$ relaxation times in the myocardium were also generated (Fig. 5c,f). Based on these histograms, the peak intra- and extracellular compartment volume fractions $\left(\mathrm{V}_{i}\right.$ and $\left.\mathrm{V}_{e}\right)$ and $\mathrm{T}_{2}$ * values were calculated for each specimen. The peak $T_{1}$ value of each compartment's distribution was then calculated. These volume fractions and relaxation properties are listed in Table 2. Using the volume fraction maps to segment out tissue voxels that were likely to contain a large proportion of small vessels, a qualitative analysis then related the magnetic susceptibility to the squared sine of the myocardial fiber angle in the resulting tissue ROI. Similar to the qualitative results in Fig. 4, the voxelwise analysis reported that the magnetic susceptibility of muscle tissue appears more diamagnetic as the myocardial fiber angle increases. For a typical specimen, this is emphasized by the linear regression fit of the mean magnetic susceptibility of the multiecho GRE data as a function of $\sin ^{2} \theta$ in Fig. 6. From the three regression fits (mean $R^{2}=0.035$ ), the estimated susceptibility anisotropy $\left(\mathrm{X}_{0^{\circ}}-\mathrm{X}_{90^{\circ}}\right)$ was $5.94 \pm$

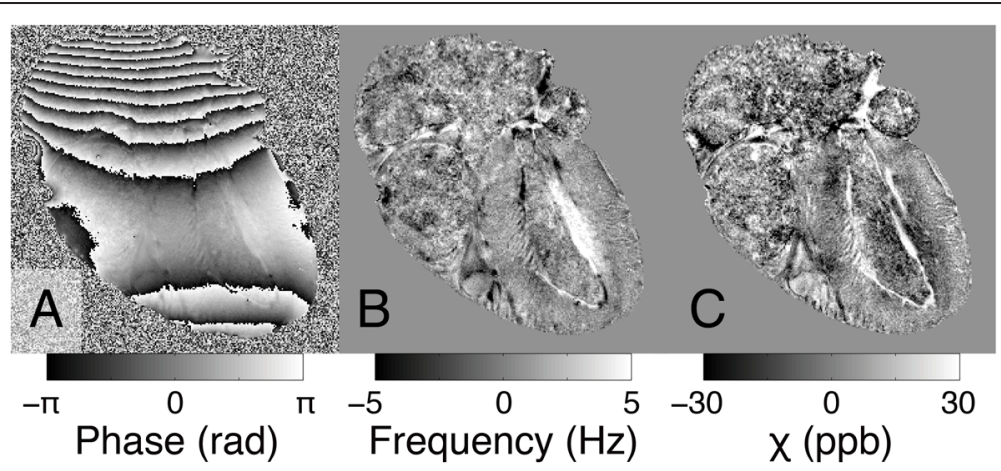

Fig. 2 Reconstruction pipeline for processing the magnetic susceptibility of the mouse heart specimens. Raw phase (a), frequency (b), and magnetic susceptibility (c) maps were generated for each echo from multi-echo gradient-recalled echo image data. Shown are examples of results at TE $=22.7 \mathrm{~ms}$ 

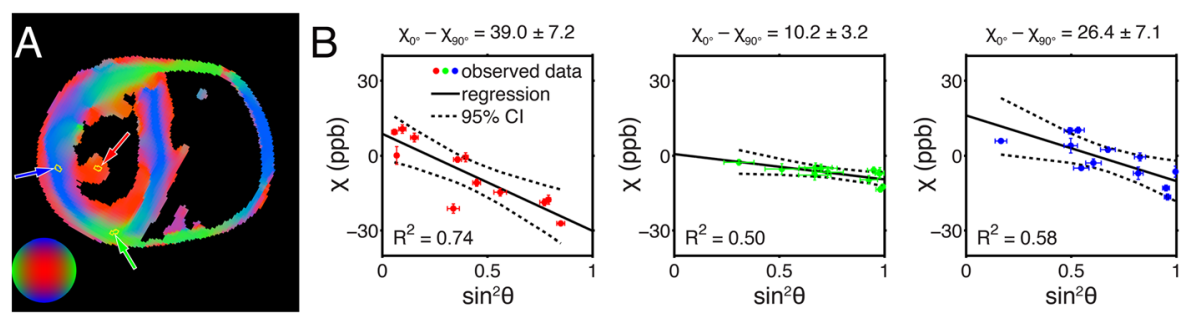

Fig. 3 Mean apparent magnetic susceptibility as a function of mean myofiber orientation in localized tissue regions. Myofiber orientation was calculated from the principal eigenvector of the DTI data (a). Each small ROI ( $n=10$ voxels) is outlined in yellow and represents myocardial fibers from one of three approximately orthogonal directions (represented by green, red, and blue). Magnetic susceptibility data were calculated from multi-orientation GRE image data and correlated with the fiber orientation. Each data point represents a measurement acquired from an individual specimen orientation. The results of the linear regression, as well as a $95 \%$ confidence interval, are given for each region (b). The susceptibility anisotropy estimates (mean \pm standard error) for each linear fit are shown. Error bars represent the standard deviations of each parameter within an individual $\mathrm{ROI}$

$0.47 \mathrm{ppb}$, and the estimated susceptibility of fieldparallel fibers $\left(\mathrm{X}_{0^{\circ}}\right)$ was $0.28 \pm 1.95 \mathrm{ppb}$. The weak correlation signifies that susceptibility anisotropy varies throughout the tissue, but is still measurably present in the heart as a whole.

\section{Model prediction of susceptibility anisotropy}

A sample image of the forward calculation of the frequency map from the susceptibility tensor-valued myocardial tissue volume array is shown in Fig. 7. Individual myofilaments within the simulated volume yield

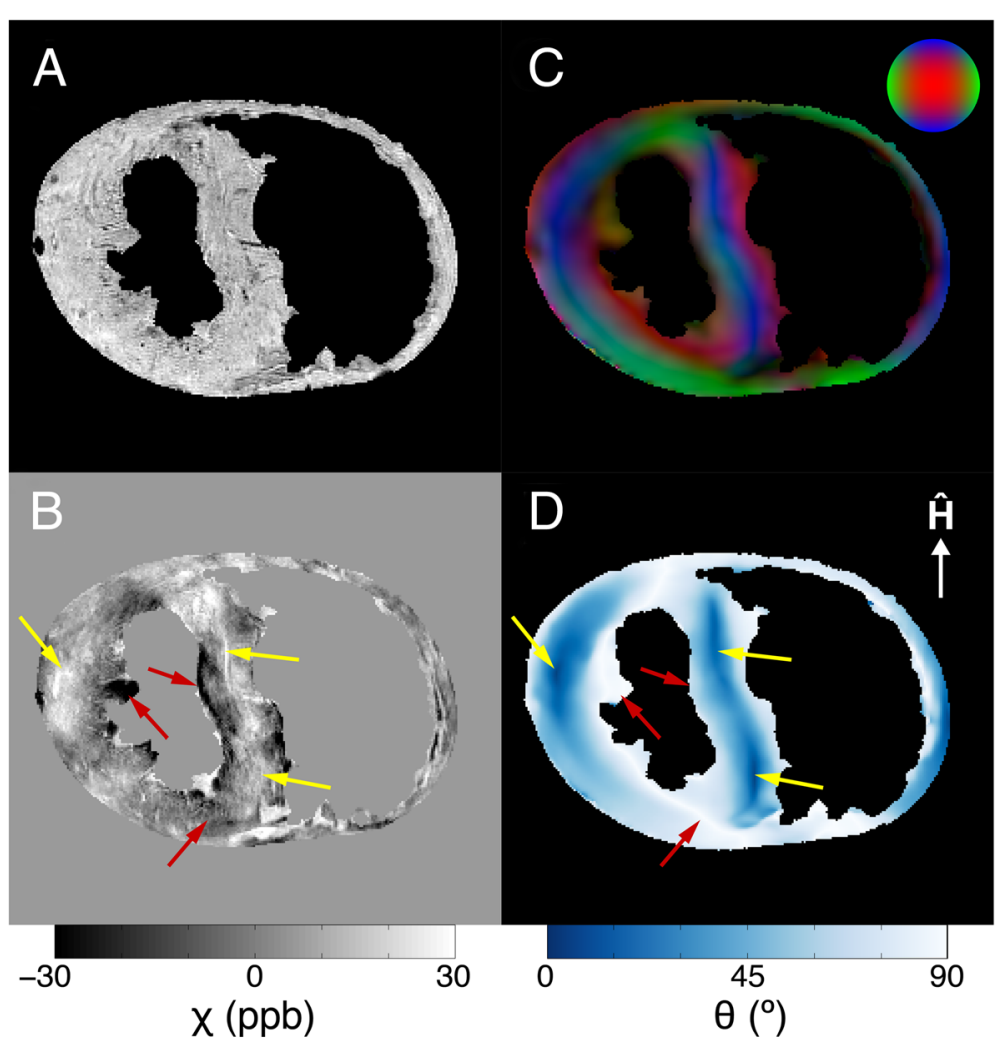

Fig. 4 Correlation between apparent magnetic susceptibility and myofiber orientation in a typical specimen. Anatomical reference image created by averaging GRE magnitude data from echoes 1-4 (a). Mean magnetic susceptibility map calculated from multi-echo GRE image phase data (b). DTI myofiber orientation map that has been weighted by the fractional anisotropy and converted into red/green/blue values, where blue indicates the magnetic field direction (c). The major eigenvector of the DTI data was used to calculate the myofiber angle relative to the magnetic field direction (d) Yellow arrows indicate myofibers that are nearly parallel to $B_{0}$ and more paramagnetic. Red arrows indicate myofibers that are nearly perpendicular to $B_{0}$ and more diamagnetic 


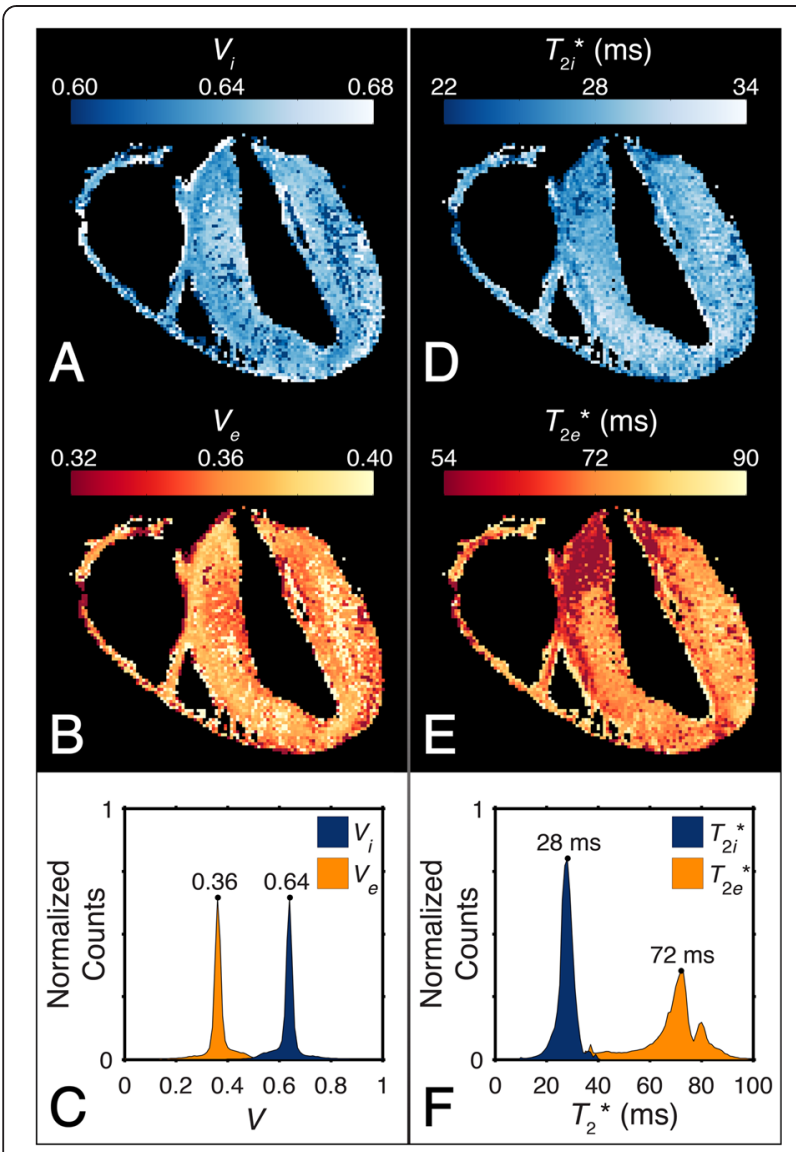

Fig. 5 Myocardium compartmentalization. The results of the voxelwise ( $n=1,515,232$ ), SNR-weighted, biexponential signal-fitting algorithm used to probe compartment volume fractions and $\mathrm{T}_{2}{ }^{*}$ values in a typical specimen are shown in the intracellular (a) and extracellular (b) volume fraction maps and their respective distributions (c). Intracellular (d) and extracellular (e) $\mathrm{T}_{2}{ }^{*}$ calculations yield $\mathrm{T}_{2}{ }^{*}$ distributions (f) with well-defined peaks. The modal volume fraction and $T_{2}{ }^{*}$ values for the two compartments are annotated within the figure with the subscripts $i$ and $e$ indicating intra- and extracellular compartments, respectively

frequency contributions that are predominantly isolated from one another. Thus, if the size of the model volume were increased to include multiple simulated volume units, measurements of susceptibility anisotropy would be consistent with a single, simulated volume unit.

Using the bulk susceptibility anisotropy data calculated from individual GRE echo images, the model computed a best-fit relative isotropic susceptibility value for the

Table 2 Intra- and extracellular relaxation properties of ex vivo myocardium (mean \pm standard deviation)

\begin{tabular}{lcc}
\hline Parameter & Intracellular & Extracellular \\
\hline Volume fraction, $n=3$ & $0.65 \pm 0.01$ & $0.35 \pm 0.01$ \\
$\mathrm{~T}_{2}{ }^{*}(\mathrm{~ms}), n=3$ & $29 \pm 1$ & $76 \pm 4$ \\
$\mathrm{~T}_{1}(\mathrm{~ms}), n=1$ & 1050 & 1750 \\
\hline
\end{tabular}

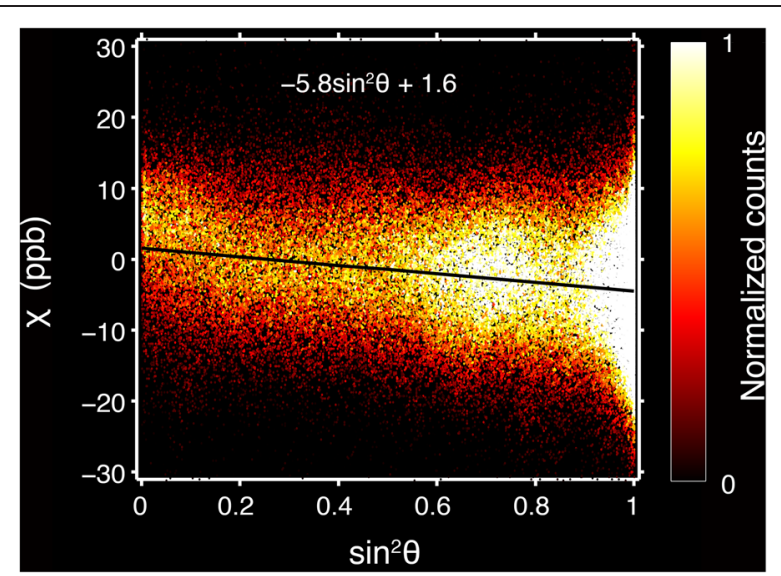

Fig. 6 Magnetic susceptibility anisotropy of myocardium. Twodimensional histogram of the voxelwise $(n=532,754)$ mean magnetic susceptibility as a function of the squared sine of the myofiber angle for a typical specimen. The average apparent magnetic susceptibility was calculated from the multi-echo GRE data acquired in a single specimen orientation. Myofiber orientation was calculated from the principal eigenvector of the DTI data

peptide group tensor $\left(X_{I}=0.04 \pm 0.01 \mathrm{ppm}\right)$. Fig. 8a compares the simulated anisotropy data to the data acquired from the mouse heart specimens using CMR. As TE increases, the susceptibility anisotropy decreases in both the simulated and acquired data. Figure $8 \mathrm{~b}$ shows that the simulated susceptibility anisotropy of the intracellular compartment decreases as TE increases even though the extracellular compartment is being ignored. This is likely due to the dephasing of spins within the volume, which intensifies as TE increases.

The results of the simulation for the different models of multi-compartment relaxation are shown in Fig. 9.

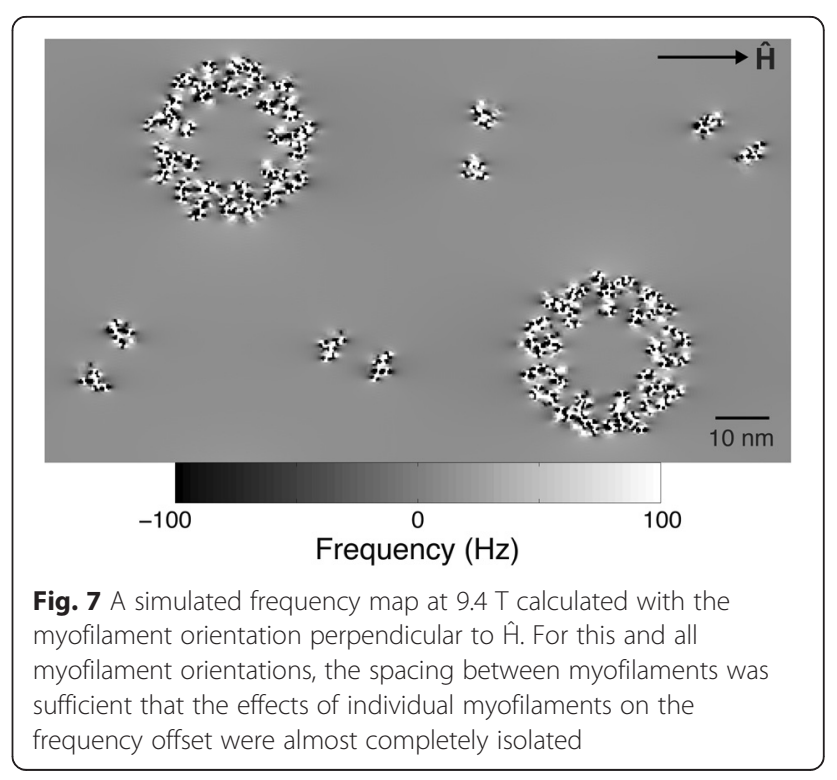



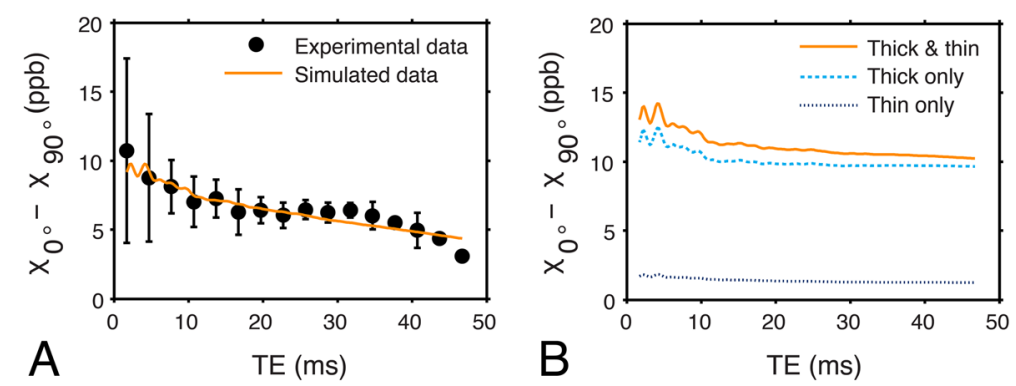

Fig. 8 Comparison between experimental and simulated susceptibility anisotropy data. The experimental data were acquired from whole mouse myocardium specimens $(n=3)$ at $9.4 \mathrm{~T}$, whereas the simulated data were calculated using the multi-filament model with two signal compartments (a). The simulated anisotropy of the intracellular compartment (b) is calculated by ignoring signal contributions from the extracellular compartment and using the fitted isotropic component $\left(X_{X}\right)$ and fixed anisotropic $\left(X_{A}\right)$ component values of the peptide group susceptibility tensor. The simulated anisotropy data are shown for when the model's intracellular compartment contains both thick and thin filaments, thick filaments only, and thin filaments only. Error bars indicate the standard deviation of the experimental data

Both multi-compartment $\mathrm{T}_{1}$ and $\mathrm{T}_{2}{ }^{*}$ relaxation have a relatively small effect on susceptibility anisotropy as a function of TE, though the fitted isotropic susceptibility value of the peptide group differs for the data that was only $\mathrm{T}_{2}{ }^{*}$ weighted $\left(\mathrm{X}_{I}=-0.8 \pm 0.1 \mathrm{ppm}\right)$ and not weighted $\left(X_{I}=0 \pm 0.1 \mathrm{ppm}\right)$. This demonstrates that, even if relaxation time estimates are only approximate, the model still reasonably simulates the experimental susceptibility anisotropy data.

To emulate the experimental data acquired from the specimen, the model applied $\mathrm{T}_{2}$ *-weighting to the simulated extracellular compartment signal magnitude to represent the effect of spin dephasing. In the intracellular compartment, however, $\mathrm{T}_{2}$-weighting is inherently modeled due to the inhomogeneous magnetic susceptibility distribution of the simulated intracellular volume. The effective simulated intracellular $\mathrm{T}_{2}$ ' value associated with the mean myocardial fiber angle of the three specimens $\left(\sim 50^{\circ}\right)$ was $\mathrm{T}_{2 i}{ }^{\prime}=40 \mathrm{~ms}$. Thus, it was necessary to superpose $\mathrm{T}_{2}$-weighting on the intracellular volume signal to effectively produce $\mathrm{T}_{2}{ }^{*}$ signal weighting. In the complete model, $\mathrm{T}_{2}$-weighting was applied to the intracellular compartment with $\mathrm{T}_{2 i}=109 \mathrm{~ms}$ in order to enforce the condition that $1 / \mathrm{T}_{2 i}{ }^{*}=1 / \mathrm{T}_{2 i}+1 / \mathrm{T}_{2 i}{ }^{\prime}$. Figure $9 \mathrm{~b}$ emphasizes the effect of erroneously replacing $\mathrm{T}_{2}$-weighting with either $\mathrm{T}_{2}{ }^{*}$-weighting, or no additional weighting on top of the inherent $\mathrm{T}_{2}$ ' signal weighting in the intracellular compartment. The fitted isotropic susceptibility for these model variations were $\mathrm{X}_{I}=-1.2 \pm 0.1$ (additional $\mathrm{T}_{2}{ }^{*}$ weighting) and $\mathrm{X}_{I}=1.5 \pm 0.1 \mathrm{ppm}$ (no additional weighting).

\section{Discussion}

\section{Multi-filament model predicts susceptibility anisotropy}

Myocardial fiber orientation plays an essential role in heart structure and function, and is frequently assessed in studies of healthy and diseased myocardium. Susceptibility imaging may potentially be used to analyze the organization and integrity of myocardial fibers by exploiting the anisotropic magnetic susceptibility of muscle tissue. The results produced by this microstructural model show that the diamagnetic anisotropy of the
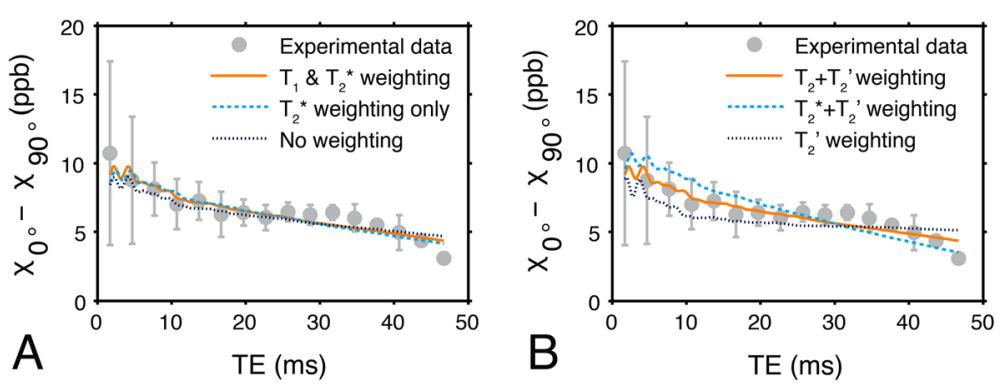

Fig. 9 Comparison between susceptibility anisotropy data simulated under different signal models. a Experimental susceptibility anisotropy and simulated susceptibility anisotropy data when the model includes $T_{1}$ and $T_{2}{ }^{*}$-weighting, $T_{2}{ }^{*}$-weighting only, and no weighting in both the intraand extracellular signal compartments. The simulated anisotropy is minimally affected by excluding multi-compartment relaxation effects from the model. $\mathbf{b}$ Experimental data from the specimen and simulated data from a model demonstrating the effect of superposing $T_{2}, T_{2}{ }^{*}$, and no additional weighting on top of the inherent intracellular $T_{2}{ }^{\prime}$ that exists due to the field inhomogeneity produced by the simulated peptide bonds. The additional intracellular spin-spin relaxation weighting significantly impacts susceptibility anisotropy as a function of TE, and is most correct in the model simulating $T_{2}{ }^{\prime}$ superposed with $T_{2}$. Error bars indicate the standard deviation of the experimental data 
polypeptide bonds in muscle filaments is potentially a major contributor to the fiber-orientation-dependent susceptibility contrast visualized using CMR. This is because the amino acids in the $\alpha$-helix are arranged with the peptide groups lying in a plane parallel to the helix axis. The summation of the individual peptide bond susceptibilities, which each have their most diamagnetic component perpendicular to the myofiber axis, produces bulk magnetic anisotropy on a scale measurable by susceptibility CMR. To simulate CMR data that exhibited susceptibility anisotropy using a model of the magnetically anisotropic $\alpha$-helix, it was necessary to calculate a fitted isotropic susceptibility value for the peptide group tensor. Magnetic susceptibility values are calculated from image frequency data that are referenced to the carrier frequency of excitation radiofrequency pulses, hence, the isotropic susceptibility component of the peptide group tensor is relative and may vary from scan to scan.

\section{Multi-compartment effects and applications}

The intra- and extracellular population fractions calculated for the short- $\mathrm{T}_{2}{ }^{*}$ and long- $\mathrm{T}_{2}{ }^{*}$ compartments (Table 2) are quite similar to the values found in other studies of excised rodent myocardium [37]. We acknowledge that not all of intracellular space is composed of the myofilament lattice. The myofibril volume fraction has been measured at $0.52 \pm 0.04$ of total perfused mouse myocardium cell volume [40]. For the purposes of maintaining a two-pool model, however, the simplifying assumption was made that the myofibril volume fraction was equal to the volume fraction of the short$\mathrm{T}_{2}$ * compartment. Based on our observations from the model volume simulation, multi-pool relaxation strongly affects susceptibility contrast and anisotropy in muscle tissue, as has been suggested by similar models for central nervous system white matter [41, 42]. This relaxation is intensified by contrast agents, which also augment susceptibility anisotropy in brain white matter [42], kidney tubules [16], and potentially the myocardium, though the underlying mechanism is unclear. Earlier studies have shown that compartmentalization of Gd-DTPA in the extracellular space of muscle tissue yields a significant $T_{2}$ reduction in extracellular water, but not intracellular water [43]. In this case, Gd may cause significant spin dephasing and signal attenuation in the extracellular compartment, leaving the anisotropic intracellular compartment to dominate the measured tissue susceptibility. Alternatively, the increased anisotropy may be induced by a local magnetic field generated by the contrast agent in the organized tissue matrix. Though the mechanisms are not yet understood, using contrast agents to enhance susceptibility anisotropy is likely to improve the feasibility of STI of the myocardium.
Applying STI to the study of animal models of myocardial disease will help verify the source(s) of myocardial susceptibility anisotropy.

In addition to contrast agents, tissue $\mathrm{pH}$ and the use of fixatives during specimen preparation may complicate analyses of multi-compartment signal relaxation. It has been shown in skeletal muscle that decreases in intracellular $\mathrm{pH}$ due to the accumulation of the end-products of anaerobic metabolism during exercise correlate with an increased intracellular $T_{2}$ relaxation time [44]. Changes to intra- or extracellular signal $\mathrm{T}_{2}$ relaxation times associated with $\mathrm{pH}$ would affect the weighting of the signal phase as described by Equation 3. The impact of this weighting would be very small, but it may explain a fraction of the decrease in the measured anisotropy at extremely long TEs. However, substantial and spatially coordinated $\mathrm{pH}$ differences are not expected in fixed tissue specimens that have soaked for days in phosphatebuffered saline solution.

Following the extraction of the heart specimen from the mouse, the tissue begins to degrade very rapidly. Due to the technical challenges of scanning fresh tissue for extended periods of time, particularly when acquiring data in multiple specimen orientations, it was determined that ex vivo data acquisition would require tissue fixation. Fixatives are known to shorten $T_{1}$ and $T_{2}$ in both nervous and cardiac tissues $[45,46]$, though the effects on $T_{2}$ are reversible by soaking the specimen in phosphate-buffered saline [46]. The simulated data suggest that changes to the $\mathrm{T}_{1}$ weighting would affect the isotropic susceptibility but have minimal impact on the susceptibility anisotropy measurements (Fig. 9a). This is especially true if the applied fixative shortens the $T_{1}$ of the intra- and extracellular signal compartments in a relatively proportional manner. Still, this is a limitation to the current study, particularly since fixative use is reserved for the preclinical domain. An alternative specimen preparation might exclude fixatives but require the inclusion of contrast agent in order to shorten the scan time and lessen the impact of tissue degradation. However, the presence of paramagnetic contrast agent in the specimen would complicate measurements of myocardial susceptibility anisotropy. This is because contrast agents like Gd-DTPA are more highly concentrated in the extracellular volume of muscle tissue [43], thereby disproportionally shortening both the longitudinal and transverse relaxation times and artificially inflating the susceptibility of the extracellular volume.

\section{Data acquisition and analysis considerations}

One goal of this study was to measure the inherent susceptibility anisotropy of myocardium, i.e., without the use of contrast agents. As a result, SNR limitations were a factor in both the DTI and GRE data acquisition and analysis. To achieve reasonable scan times, the DTI data 
were acquired at a lower resolution, $180 \mu \mathrm{m}$, relative to the GRE data resolution, $45 \mu \mathrm{m}$ (see Methods). Myocardial tissue is laminar, and has a typical tissue layer width of $100 \mu \mathrm{m}$. As a result, some partial voluming of the diffusion signal is expected. However, because the orientation of myocardial tissue fibers varies smoothly from layer to layer, the interpolated DTI data reasonably describe myofiber orientations in the heart at the higher resolution (Fig. 4). As for the GRE data, the first two echo images from each specimen have very low phase $\mathrm{SNR}$, which is expected when $\mathrm{TE}<<\mathrm{T}_{2}{ }^{*}$ [47]. Measurements of myocardial susceptibility anisotropy at short TEs vary greatly, which explains the data acquired at TE $<5 \mathrm{~ms}$ (Fig. 8).

Another goal of this study was to measure the average magnetic susceptibility anisotropy using data from a single specimen orientation. This estimation, however, did not quantify the tensor-valued susceptibility on a voxel basis and might be confounded by the spatial heterogeneity of myofibers. Unlike the multi-orientation data acquisition, which allowed for the analysis of the same tissue regions in multiple orientations, a singleorientation measurement protocol required correlating susceptibility contrast with fiber orientation from all different regions of the heart. As a result, measurements of anisotropic susceptibility may be affected by the selected specimen orientation. For example, when the heart specimen is oriented with its long axis parallel to the magnetic field, there are likely to be more myocardial fibers that are perpendicular to the magnetic field compared to when the long axis of the heart is perpendicular to the field direction-as it was oriented in this study. Furthermore, bulk magnetic susceptibility shifts due to the presence of spatially varying concentrations of electrolytes, proteins, and other substances with both isotropic and anisotropic susceptibility may yield fluctuations in the single-orientation anisotropy values. Especially large concentrations of diamagnetic electrolytes such as calcium decrease the bulk magnetic susceptibility of that particular tissue region, and have been shown to yield both image phase fluctuations in the brain [12] and decreased signal intensity in fluid-filled phantoms [48]. In a study of the dog heart, concentrations of calcium and other electrolytes in myocardial tissue vary only mildly between the septum and the left ventricle (LV) and right ventricle (RV) walls, as described in Table 3 [49]. Such disparities would result in small bulk isotropic susceptibility differences $(<1 \mathrm{ppb})$ between these two regions and are not sufficient to explain the observed susceptibility variation. Nevertheless, in diseased hearts, large decreases in bulk magnetic susceptibility in different tissue regions may be indicative of pathologies associated with abnormal levels of electrolytes, e.g., calcium and iron deposition. Hence, susceptibility and susceptibility anisotropy may potentially be sensitive to diseases correlated with calcium elevation such as cardiac hypertrophy [50].

\section{Other potential sources of anisotropy}

The peptide bonds of axially aligned myosin and tropomyosin $\alpha$-helices, though a significant source of diamagnetic anisotropy, may not act alone in producing orientationdependent susceptibility contrast in the myocardium. Other potential sources of anisotropy include actin, collagen, hemoglobin, myoglobin, and lipids. Actin represents $25 \%$ of myofilament proteins in normal rodent myocardium (myosin and tropomyosin represent about $50 \%$ and $15 \%$, respectively) [51]. Both globular and filamentous actin forms contain $\alpha$-helix structures. However, the $\alpha$ helices within actin exist in a wide range of orientations relative to the filament axis [34], and birefringence measurements show that actin only weakly aligns to an applied magnetic field [2]. Collagen, another structural protein, may also influence the observed susceptibility anisotropy of the myocardium. Due to the orientation of its peptide bonds, collagen exhibits susceptibility anisotropy that is both directionally opposite and half as strong as that of polypeptide $\alpha$-helices [18]. However, because collagen constitutes such a small fraction of rodent myocardium, $<2 \mathrm{mg} / \mathrm{g}$ [52], relative to myofibrillar proteins, 41.5

Table 3 Estimated bulk magnetic susceptibility shift differences due to electrolytes in myocardial tissue

\begin{tabular}{|c|c|c|c|c|}
\hline \multirow[t]{2}{*}{ Electrolyte } & $\begin{array}{l}\text { Molar susceptibility } \\
\left.\text { (CGS, } \mathrm{cm}^{3} / \mathrm{mol}\right)[68]\end{array}$ & $\begin{array}{l}\text { LV concentration } \\
\left(\mathrm{CGS}, \mathrm{mol} / \mathrm{cm}^{3}\right)[49]\end{array}$ & $\begin{array}{l}\text { RV concentration } \\
\left(\mathrm{CGS}, \mathrm{mol} / \mathrm{cm}^{3}\right)[49]\end{array}$ & $\begin{array}{l}\text { LV-RV Volume susceptibility } \\
\text { difference (SI, unitless) }\end{array}$ \\
\hline & $x_{m} \times 10^{-6}$ & $c \times 10^{-6}$ & $c \times 10^{-6}$ & $x_{v}, \mathrm{ppb}$ \\
\hline $\mathrm{Ca}^{2+}$ & -10.4 & $0.80 \pm 0.10$ & $0.90 \pm 0.27$ & 0.01 \\
\hline $\mathrm{Cl}^{-}$ & -23.4 & $29.07 \pm 2.03$ & $31.53 \pm 2.94$ & 0.72 \\
\hline $\mathrm{K}^{+}$ & -14.9 & $91.04 \pm 4.93$ & $88.78 \pm 5.64$ & -0.42 \\
\hline $\mathrm{Mg}^{2+}$ & -5.0 & $10.49 \pm 1.49$ & $10.07 \pm 1.58$ & -0.03 \\
\hline $\mathrm{Na}^{+}$ & -6.8 & $32.97 \pm 1.60$ & $34.94 \pm 5.64$ & 0.17 \\
\hline
\end{tabular}

The volume susceptibility differences between the LV and RV walls due to electrolytes are very small compared to the observed susceptibility anisotropy

${ }^{a}$ Magnetic susceptibility values for these electrolytes are typically reported in the literature as molar susceptibilities using CGS units

${ }^{b}$ Calculated from dog myocardium data and the density of fat-free myocardial tissue, $1.054 \mathrm{~g} / \mathrm{cm}^{3}[69]$

${ }^{c}$ Conversion from molar to volume susceptibility follows $x_{v}=x_{m}$ c. Conversion from CGS to SI units follows $X_{v}(S I)=4 \pi X_{v}(C G S)$ 
$\pm 1.1 \mathrm{mg} / \mathrm{g}$ [53], the susceptibility anisotropy of the thick and thin filament proteins appears to be more dominant.

Hemoglobin and myoglobin may have an effect on myocardial susceptibility anisotropy measurements if the distribution of these proteins in the tissue is nonuniform. For instance, if greater concentrations of paramagnetic deoxyhemoglobin exist in tissue regions with more parallel myofibers, it may increase the measured susceptibility anisotropy. To mitigate any possible effects due to deoxyhemoglobin, the myocardium was extensively perfused with a heparin/saline solution to flush blood out of the tissue during specimen preparation (see Methods). Deoxymyoglobin is a paramagnetic globular protein with a large molar susceptibility anisotropy of $-9.73 \pm 0.38 \times 10^{-4} \mathrm{~cm}^{3} / \mathrm{mol}$ [54]. However, myoglobin molecules are not structurally oriented in tissue since they are mobile and diffuse [55] - sliding past one another with little frictional interaction [56] -so collectively they do not produce susceptibility anisotropy, but rather a bulk magnetic susceptibility shift dependent on the mean susceptibility value $\left(1.35 \times 10^{-2} \mathrm{~cm}^{3} / \mathrm{mol}\right.$ [54] $)$ and local concentration of myoglobin. In human myocardium, myoglobin content within the LV is quite consistent (including the septum and posterior, lateral, and anterior walls), but greater in the LV $(2.4 \mathrm{mg} / \mathrm{g}$ wet tissue) than the RV $(1.9 \mathrm{mg} / \mathrm{g})$ [57]. Similar to the case of electrolytes (Table 3), the resulting bulk susceptibility difference between the LV and RV walls due to myoglobin is very small, an estimated $0.4 \mathrm{ppb}$, and is not sufficient to explain the observed susceptibility variation.

Finally, the lipids that form the sarcoplasmic reticulum surrounding individual myofibrils may be a source of susceptibility anisotropy. Lipids are known to be more diamagnetic in the direction parallel to their chain structure, and are believed to be a major source of anisotropy in brain white matter. In rodents, however, the lipid volume fraction is 0.16 [58] in brain white matter and only $\sim 0.03$ in myocardium cells [59]. It is worth noting that the cell volume fraction of myofibrillar mass is $\sim 0.45-0.47$ in rodent myocardium $[59,60]$, further suggesting that that the known anisotropy of myofibrillar peptide bonds is a major contributor, and most likely the dominant contributor, to the orientation-dependent susceptibility contrast visualized with CMR.

\section{Conclusions}

Susceptibility imaging may potentially be used to determine the organization and integrity of myofibers by probing the anisotropic magnetic susceptibility of myocardial tissue. The multi-filament model demonstrates that the arrangement of the diamagnetically anisotropic peptide bonds forming these myofibers contributes substantially to the bulk susceptibility anisotropy of the heart. Greater understanding of the mechanisms generating this anisotropy may lead to improved techniques for studying models of healthy and diseased myocardium.

\section{Abbreviations}

CMR: Cardiovascular magnetic resonance; DTI: Diffusion tensor imaging; GRE: Gradient-recalled echo; LV: Left ventricle; ROI: Region of interest; RV: Right ventricle; SE: Spin echo; SNR: Signal-to-noise ratio; STI: Susceptibility tensor imaging; TE: Echo time; TR: Repetition time.

\section{Competing interests}

The authors declare that they have no competing interests.

\section{Authors' contributions}

All authors participated in the design of the study. YQ and RD carried out the ex-vivo fixation preparation of the mouse myocardium specimens. RD performed the MR image acquisitions, carried out the data reconstruction and processing, developed the simulation, performed the computational analyses, and drafted the manuscript. $\mathrm{RD}$ and $\mathrm{CL}$ were involved in the conception of the simulation and the interpretation of experimental and simulated data. All authors participated in either the design and drafting of the manuscript or its critical revision for important intellectual input. All authors also read and approved the final manuscript.

\section{Acknowledgments}

We thank G. Allan Johnson, PhD, Gary Cofer, MS, and Luke Xie, PhD, for their assistance in this study and Sally Zimney for her help in preparing the manuscript. All imaging was carried out at the Center for In Vivo Microscopy of Duke University. This study was supported in part by the National Institutes of Health through NIBIB P41 EB015897, NIBIB T32 EB001040, NIMH R01 MH096979, and NHLBI R21 HL122759, and by the National Multiple Sclerosis Society through grant RG4723.

\section{Author details}

${ }^{1}$ Center for In Vivo Microscopy, Duke University Medical Center, Box 3302, Durham, NC 27710, USA. ${ }^{2}$ Biomedical Engineering, Duke University Medical Center, Campus Box 90281, Durham, NC 27708, USA. ${ }^{3}$ Brain Imaging \& Analysis Center, Duke University Medical Center, Box 3918, Durham, NC 27710, USA. ${ }^{4}$ Radiology, Duke University Medical Center, Box 3808, Durham, NC 27710, USA.

Received: 19 December 2014 Accepted: 23 June 2015

Published online: 16 July 2015

\section{References}

1. Arnold W, Steele R, Mueller H. On the magnetic asymmetry of muscle fibers. Proc Natl Acad Sci USA. 1958:44:1-4.

2. Torbet J, Dickens MJ. Orientation of skeletal muscle actin in strong magnetic fields. FEBS Lett. 1984;173:403-6.

3. Bovendeerd PHM, Arts T, Huyghe JM, van Campen DH, Reneman RS Dependence of local left ventricular wall mechanics on myocardial fiber orientation: A model study. J Biomech. 1992;25:1129-40.

4. Tezuka F. Muscle fiber orientation in normal and hypertrophied hearts. Tohoku J Exp Med. 1975;117:289-97.

5. Wickline SA, Verdonk ED, Wong AK, Shepard RK, Miller JG. Structural remodeling of human myocardial tissue after infarction. Quantification with ultrasonic backscatter. Circulation. 1992:85:259-68.

6. Strijkers GJ, Bouts A, Blankesteijn WM, Peeters TH, Vilanova A, van Prooijen $M C$, et al. Diffusion tensor imaging of left ventricular remodeling in response to myocardial infarction in the mouse. NMR Biomed. 2009;22:182-90.

7. Fenton F, Karma A. Vortex dynamics in three-dimensional continuous myocardium with fiber rotation: Filament instability and fibrillation. Chaos (Woodbury, NY). 1998:8:20-47.

8. Hsu EW, Muzikant AL, Matulevicius SA, Penland RC, Henriquez CS. Magnetic resonance myocardial fiber-orientation mapping with direct histological correlation. Am J Physiol. 1998:274:H1627-34.

9. Scollan DF, Holmes A, Winslow R, Forder J. Histological validation of myocardial microstructure obtained from diffusion tensor magnetic resonance imaging. Am J Physiol. 1998;275:H2308-18.

10. Sosnovik DE, Mekkaoui C, Huang S, Chen HH, Dai G, Stoeck CT, et al. Microstructural impact of ischemia and bone marrow-derived cell therapy 
revealed with diffusion tensor magnetic resonance imaging tractography of the heart in vivo. Circulation. 2014;129:1731-41.

11. Köhler S, Hiller K-H, Waller C, Jakob PM, Bauer WR, Haase A. Visualization of myocardial microstructure using high-resolution $\mathrm{T}^{*}$ imaging at high magnetic field. Magn Reson Med. 2003;49:371-5.

12. Yamada N, Imakita S, Sakuma T, Takamiya M. Intracranial calcification on gradient-echo phase image: depiction of diamagnetic susceptibility. Radiology. 1996;198:171-8.

13. Liu C. Susceptibility tensor imaging. Magn Reson Med. 2010;63:1471-7.

14. Liu C, Li W. Imaging neural architecture of the brain based on its multipole magnetic response. Neuroimage. 2013;67:193-202.

15. Lee J, Shmueli K, Fukunaga M, van Gelderen P, Merkle H, Silva AC, et al. Sensitivity of MRI resonance frequency to the orientation of brain tissue microstructure. Proc Natl Acad Sci USA. 2010;107(11):5130-5.

16. Xie L, Dibb R, Cofer GP, Li W, Nicholls PJ, Johnson GA, et al. Susceptibility tensor imaging of the kidney and its microstructural underpinnings. Magn Reson Med. 2014;73:1270-81.

17. Pauling L, Corey RB, Branson HR. The structure of proteins; two hydrogenbonded helical configurations of the polypeptide chain. Proc Natl Acad Sci USA. 1951;37:205-11.

18. Worcester DL. Structural origins of diamagnetic anisotropy in proteins. Proc Natl Acad Sci USA. 1978;75:5475-7.

19. Pauling L. Diamagnetic anisotropy of the peptide group. Proc Natl Acad Sci USA. 1979;76:2293-4.

20. Angeli S, Befera N, Peyrat J-M, Calabrese E, Johnson GA, Constantinides C. A high-resolution cardiovascular magnetic resonance diffusion tensor map from ex-vivo C57BL/6 murine hearts. J Cardiovasc Magn Reson. 2014;16:77.

21. Li W, Avram AV, Wu B, Xiao X, Liu C. Integrated Laplacian-based phase unwrapping and background phase removal for quantitative susceptibility mapping. NMR Biomed. 2014;27:219-27.

22. Salomir R, de Senneville BD, Moonen CTW. A fast calculation method for magnetic field inhomogeneity due to an arbitrary distribution of bulk susceptibility. Concepts Magn Reson B. 2003;19B:26-34.

23. Shmueli K, de Zwart JA, van Gelderen P, Li T-Q, Dodd SJ, Duyn JH. Magnetic susceptibility mapping of brain tissue in vivo using MRI phase data. Magn Reson Med. 2009:62:1510-22.

24. Liu T, Spincemaille P, de Rochefort L, Kressler B, Wang Y. Calculation of susceptibility through multiple orientation sampling (COSMOS): A method for conditioning the inverse problem from measured magnetic field map to susceptibility source image in MRI. Magn Reson Med. 2009:61:196-204.

25. de Rochefort L, Liu T, Kressler B, Liu J, Spincemaille P, Lebon V, et al. Quantitative susceptibility map reconstruction from MR phase data using bayesian regularization: validation and application to brain imaging. Magn Reson Med. 2010;63:194-206.

26. Wu B, Li W, Guidon A, Liu C. Whole brain susceptibility mapping using compressed sensing. Magn Reson Med. 2012;67:137-47.

27. Li W, Wu B, Liu C. Quantitative susceptibility mapping of human brain reflects spatial variation in tissue composition. Neuroimage. 2011;55:1645-56.

28. Wang R, Benner T, Sorensen AG, Weeden VJ. Diffusion Toolkit: A Software Package for Diffusion Imaging Data Processing and Tractography. In: Proceedings of the 15th Annual ISMRM, Berlin, Germany. 2007. p. 3720.

29. Yushkevich PA, Piven J, Hazlett HC, Smith RG, Ho S, Gee JC, et al. User-guided 3D active contour segmentation of anatomical structures: significantly improved efficiency and reliability. Neuroimage. 2006;31:1116-28.

30. Kabsch W, Mannherz HG, Suck D. Three-dimensional structure of the complex of actin and DNase I at 4.5 A resolution. EMBO J. 1985;4:2113-8.

31. Ashton FT, Weisel J, Pepe FA. The myosin filament XIV backbone structure. Biophys J. 1992;61:1513-28.

32. Pepe FA, Ashton FT, Street C, Weisel J. The myosin filament. X. Observation of nine subfilaments in transverse sections. Tissue Cell. 1986;18:499-508.

33. Tanner BCW, Daniel TL, Regnier M. Sarcomere Lattice Geometry Influences Cooperative Myosin Binding in Muscle. PLoS Comput Biol. 2007;3:e115.

34. Kabsch W, Mannherz HG, Suck D, Pai EF, Holmes KC. Atomic structure of the actin: DNase I complex. Nature. 1990;347:37-44.

35. Luna A, Amekraz B, Tortajada J, Morizur JP, Alcamí M, Mó O, et al. Modeling the Interactions between Peptide Functions and $\mathrm{Cu}(\mathrm{l})$ : Formamide - $\mathrm{Cu}+$ Reactions in the Gas Phase. J Am Chem Soc. 1998;120:5411-26.

36. Tigelaar $\mathrm{HL}$, Flygare WH. Molecular zeeman effect in formamide and the alpha-proton chemical shift in poly(L-alanine). J Am Chem Soc. 1972:94:343-6.
37. Nordhoy W, Anthonsen HW, Bruvold M, Brurok H, Skarra S, Krane J, et al. Intracellular manganese ions provide strong $\mathrm{T1}$ relaxation in rat myocardium. Magn Reson Med. 2004;52:506-14.

38. Aliev MK, Dos Santos P, Hoerter JA, Soboll S, Tikhonov AN, Saks VA. Water content and its intracellular distribution in intact and saline perfused rat hearts revisited. Cardiovasc Res. 2002;53:48-58.

39. Chu SC, Xu Y, Balschi JA, Springer CS. Bulk magnetic susceptibility shifts in NMR studies of compartmentalized samples: use of paramagnetic reagents. Magn Reson Med. 1990;13:239-62.

40. Barth E, Stämmler G, Speiser B, Schaper J. Ultrastructural quantitation of mitochondria and myofilaments in cardiac muscle from 10 different animal species including man. J Mol Cell Cardiol. 1992;24:669-81.

41. Wharton S, Bowtell R. Fiber orientation-dependent white matter contrast in gradient echo MRI. Proc Natl Acad Sci USA. 2012;109:18559-64.

42. Dibb R, Li W, Cofer G, Liu C. Microstructural origins of gadolinium-enhanced susceptibility contrast and anisotropy. Magn Reson Med. 2014;72:1702-11.

43. Adzamli IK, Jolesz FA, Bleier AR, Mulkern RV, Sandor T. The effect of gadolinium DTPA on tissue water compartments in slow- and fast-twitch rabbit muscles. Magn Reson Med. 1989;11:172-81.

44. Damon BM, Gregory CD, Hall KL, Stark HJ, Gulani V, Dawson MJ. Intracellular acidification and volume increases explain R2 decreases in exercising muscle. Magn Reson Med. 2002;47:14-23.

45. Carpenter JP, He T, Kirk P, Roughton M, Anderson LJ, de Noronha SV, et al. Calibration of myocardial T2 and T1 against iron concentration. J Cardiovasc Magn Reson. 2014;16:62

46. Shepherd TM, Thelwall PE, Stanisz GJ, Blackband SJ. Aldehyde fixative solutions alter the water relaxation and diffusion properties of nervous tissue. Magn Reson Med. 2009;62:26-34.

47. Wu B, Li W, Avram AV, Gho SM, Liu C. Fast and tissue-optimized mapping of magnetic susceptibility and $2^{*}$ with multi-echo and multi-shot spirals. Neuroimage. 2012;59:297-305.

48. Kucharczyk W, Henkelman RM. Visibility of calcium on MR and CT: can MR show calcium that CT cannot? Am J Neuroradiol. 1994;15:1145-8.

49. Moulder PV, Eichelberger L, Rams JJ, Greenburg AG. Water, nitrogen, and electrolyte content of right and left ventricular walls and interventricular septum of normal canine hearts. Circ Res. 1966;19:662-7.

50. Marks AR. Calcium and the heart: a question of life and death. J Clin Invest. 2003:111:597-600

51. Drott C, Lonnroth C, Lundholm K. Protein synthesis, myosin ATPase activity and myofibrillar protein composition in hearts from tumour-bearing rats and mice. Biochem J. 1989;264:191-8.

52. Bradshaw AD, Baicu CF, Rentz TJ, Van Laer AO, Bonnema DD, Zile MR. Age-dependent alterations in fibrillar collagen content and myocardial diastolic function: role of SPARC in post-synthetic procollagen processing. Am J Physiol Heart Circ Physiol. 2010;298:H614-22.

53. Hunter RJ, Patel VB, Miell JP, Wong HJ, Marway JS, Richardson PJ, et al. Diarrhea reduces the rates of cardiac protein synthesis in myofibrillar protein fractions in rats in vivo. J Nutr. 2001;131:1513-9.

54. Bertini I, Luchinat C, Turano P, Battaini G, Casella L. The Magnetic Properties of Myoglobin as Studied by NMR Spectroscopy. Chemistry. 2003;9:2316-22.

55. Gros G, Wittenberg BA, Jue T. Myoglobin's old and new clothes: from molecular structure to function in living cells. J Exp Biol. 2010;213:2713-25.

56. Riveros-Moreno V, Wittenberg JB. The Self-Diffusion Coefficients of Myoglobin and Hemoglobin in Concentrated Solutions. J Biol Chem. 1972;247:895-901.

57. Swaanenburg JC, Visser-VanBrummen PJ, DeJongste MJ, Tiebosch AT. The content and distribution of troponin I, troponin T, myoglobin, and alpha-hydroxybutyric acid dehydrogenase in the human heart. Am J Clin Pathol. 2001;115:770-7.

58. Naito A, Nagao T, Obata M, Shindo Y, Okamoto M, Yokoyama S, et al. Dynorphin induced magnetic ordering in lipid bilayers as studied by (31)P NMR spectroscopy. Biochim Biophys Acta. 2002;1558:34-44.

59. Sachs HG, Colgan JA, Lazarus ML. Ultrastructure of the aging myocardium: A morphometric approach. Am J Anat. 1977;150:63-71.

60. Wittenberg JB, Wittenberg BA. Myoglobin function reassessed. J Exp Biol. 2003:206:2011-20.

61. Kensler RW. The mammalian cardiac muscle thick filament: Crossbridge arrangement. J Struct Biol. 2005;149:303-12.

62. Pollard TD. Structure and polymerization of Acanthamoeba myosin-II filaments. J Cell Biol. 1982;95:816-25. 
63. Atkinson SJ, Stewart M. Molecular basis of myosin assembly: coiled-coil interactions and the role of charge periodicities. J Cell Sci Suppl. 1991;14:7-10.

64. Fischman DA. An electron microscope study of myofibril formation in embryonic chick skeletal muscle. J Cell Biol. 1967;32:557-75.

65. McLachlan AD, Stewart M. The 14-fold periodicity in a-tropomyosin and the interaction with actin. J Mol Biol. 1976;103:271-98.

66. Gunning P, editor. Tropomyosin. Austin, TX: Springer Science + Business Media; 2008. New York, NY; Landes Bioscience.

67. Pauling L, Corey RB. Compound Helical Configurations of Polypeptide Chains: Structure of Proteins of the [alpha]-Keratin Type. Nature. 1953:171:59-61.

68. Likhtenshtein GI, Yamauchi J, Nakatsuji S, Tamura R, Smirnov Al. Nitroxides Applications in Chemistry, Biomedicine, and Materials Science. Hoboken: Wiley-VCH Imprint, John Wiley \& Sons, Incorporated; 2008.

69. Allen TH, Krzywicki HJ, Roberts JE. Density, fat, water and solids in freshly isolated tissues. J Appl Physiol. 1959;14:1005-8.

\section{Submit your next manuscript to BioMed Central and take full advantage of:}

- Convenient online submission

- Thorough peer review

- No space constraints or color figure charges

- Immediate publication on acceptance

- Inclusion in PubMed, CAS, Scopus and Google Scholar

- Research which is freely available for redistribution 